\title{
Crystallography of SARS-CoV-2 Non-structural Proteins
}

\author{
A Joachimiak ${ }^{1}$, C Chang ${ }^{2}$, M Endres ${ }^{3}$, R Jedrzejczak ${ }^{3}$, Y Kim ${ }^{4}, \mathrm{~N}_{\text {Maltseva }}{ }^{3}, \mathrm{~K}_{\text {Michalska }}{ }^{1}$, J \\ Osipiuk $^{3}$, L Stols ${ }^{3}, \mathrm{~K} \mathrm{Tan}^{5}, \mathrm{C}$ Tesar $^{3}, \mathrm{M} \mathrm{Wilamowski}^{3}$ \\ ${ }^{1}$ Argonne National Laboratory, ${ }^{2}$ Center for Structural Genomics of Infectious Diseases, University \\ of Chicago, ${ }^{3}$ Center for Structural Genomics of Infectious Diseases, ${ }^{4}$ Argonne National Lab, \\ Lemont, IL, ${ }^{5}$ Argonne National Laboratory, Argonne, IL \\ andrzejj@anl.gov
}

The coronavirus SARS-CoV-2 is an agent causing COVID-19 pandemic affecting millions of people. At present there is no effective vaccine or proven drug to prevent infections and stop virus proliferation. Although the virus is similar to SARS- and MERS-CoVs, the detailed information about SARS-CoV-2 proteins structures and functions is urgently needed to rapidly develop effective therapeutics. We have applied high-throughput protein production and structure determination pipeline at the Center for Structural Genomics of Infectious Diseases to produce SARSCoV-2 proteins and determine high resolution crystals structures. We have focused on nonstructural proteins (Nsps) expressed as polyproteins $1 \mathrm{a}$ and $1 \mathrm{ab}$ that are processed and assemble into a large membrane-bound replicasetranscriptase complex exhibiting multiple enzymatic and binding activities. Thus far we have determined 28 structures for $10 \mathrm{CoV}-2$ proteins. These structures include Nsp3 ADP-ribose phosphatase domain (ADRP, also known as macrodomain) and PLpro papain-like protease, Nsp5 main protease Mpro, Nsp7/Nsp8 primase complex, Nsp9 RNA-binding protein, Nsp10/Nsp16 2'-O-ribose methyltransferase complex and Nsp15 uridylate-specific endoribonuclease. We compare these structures with previously reported homologs from SARS and MERS coronaviruses and point to similarities and differences. We have also determined structures of complexes with ligands and inhibitors, including FDA approved drugs. We deposit all structures to the Protein Data Bank and release the coordinates to scientific community prior to publication. We also share all reagents and protocols. These structures provide basis for structure-based drug development.

Acta Cryst. (2020). A76, a217 